\title{
Lattice models for Non-Fermi Liquids with Tunable Transport Scalings
}

\author{
Xiao-Chuan $\mathrm{Wu},{ }^{1}$ Chao-Ming Jian, ${ }^{2,3}$ and Cenke $\mathrm{Xu}^{1}$ \\ ${ }^{1}$ Department of Physics, University of California, Santa Barbara, CA 93106, USA \\ ${ }^{2}$ Kavli Institute of Theoretical Physics, Santa Barbara, CA 93106, USA \\ 3 Station Q, Microsoft Research, Santa Barbara, California 93106-6105, USA
}

(Dated: February 28, 2019)

\begin{abstract}
A variety of exotic non-fermi liquid (NFL) states have been observed in many condensed matter systems, with different scaling relations between transport coefficients and temperature. The "standard" approach to studying these NFLs is by coupling a Fermi liquid to quantum critical fluctuations, which potentially can drive the system into a NFL. In this work we seek for an alternative understanding of these various NFLs in a unified framework. We first construct two "elementary" randomness-free models with four-fermion interactions only, whose many properties can be analyzed exactly in certain limit just like the Sachdev-Ye-Kitaev (SYK) model. The most important new feature of our models is that, the fermion scaling dimension in the conformal invariant solution in the infrared limit is tunable by charge density. Then based on these elementary models, we propose two versions of lattice models with four fermion interactions which give us non-fermi liquid behaviors with DC resistivity scaling $\varrho \sim T^{\alpha}$ in a finite temperature window, and $\alpha \in[1,2)$ depends on the fermion density in the model, which is a rather universal feature observed in many experimental systems.
\end{abstract}

PACS numbers:

\section{INTRODUCTION}

Non-fermi liquid (NFL) states represent a family of exotic metallic states that do not have long-lived quasiparticles, and hence behave fundamentally differently from the standard Landau Fermi liquid theory 1 13. The most well-known NFL, the "strange metal" phase at the optimal doping of the cuprate high temperature superconductors, has a universal scaling of its DC resistivity $\varrho \sim T^{14}[18$, while the standard Fermi liquid theory predicts $\varrho \sim T^{2}$. Recently the same strange metal behavior was observed in twisted bilayer graphene above the superconductor phase ${ }^{19}$. A consensus of the nature of the strange metal phase has not been reached yet, but a series of toy models, despite their relatively unnatural forms, seem to capture many of the key universal features of the strange metal phase. These models are the so-called Sachdev-Ye-Kitaev (SYK) model and its generalizations 20 27. For example, it was found that the SYK model has marginally relevant "pairing instability" just like the ordinary Fermi liquid state $28 \mid 29$, which is consistent with the fact that the non-Fermi liquid phase is often preempted by a dome of "ordered phase" with pair condensate of fermions (superconductivity) at low temperature 30 36. Thus the "SYK phase" can be viewed as a candidate parent phase of superconductor. Also, the recently observed anomalous charge density fluctuation of the strange meta ${ }^{37}$ suggests connection to the SYK mode 20. Last but not least, a series of generalizations based on the SYK model has shown linear- $T$ resistivity for a large temperature window, and the scaling dimension of the fermion operators in the SYK model is the key for the linear- $T$ scaling of the resistivity 38 41]. But these models, in order to ensure solubility, require fully random four-fermion interactions with a Gaussian distri- bution and zero mean, which is unlikely to exist in real materials. More recently a model on the square lattice without random interaction was constructed ${ }^{42}$, which in the soluble limit mimics the physics of the so called threeindex tensor model $25 \mid 26 / 43$, and gives us the same desirable physics such as linear- $T$ scaling of DC resistivity, and marginally relevant instability towards superconductor and other competing phases.

Most of the previously discussed generalizations of the SYK model aimed at constructing the strange metal phase with precisely linear $-T$ scaling of resistivity. But NFL can have much richer physics than the strange metal. In various systems with NFL behaviors, the DC resistivity can scale with temperature as $\varrho \sim T^{\alpha}$ with $1 \leq \alpha<2^{44} 49$, and $\alpha$ is usually tunable by varying the charge density. As we mentioned in the previous paragraph, the linear $-T$ scaling of the DC resistivity is a direct consequence of the scaling dimension $\Delta_{f}=1 / 4$ of the fermion operator in the SYK model after disorder average. To design a model with $\alpha$ between 1 and 2 , we can in principle start with the $\mathrm{SYK}_{q}$ model with $q>4$. But these models require a $q$-body interactions between the fermions, and hence are also not realistic for condensed matter systems. Thus to construct a relatively realistic NFL with $\varrho \sim T^{\alpha}$ and an arbitrary $\alpha \in[1,2)$, we need to start with a model with four-fermion interaction only and no randomness, but with conformal solutions whose fermion scaling dimensions can be different from $1 / 4$. And most ideally the fermion scaling dimension is tunable with charge density.

The standard approach of understanding these NFLs is by coupling the Fermi liquid state to a fluctuating bosonic quantum critical mode, and the relevant boson-fermion coupling can potentially drive the system into a NFL 13 . And the transport-temperature scaling would depend on 
the spatial dimensionality and also the momentum carried by the quantum critical mode. In this paper we take a different approach. We will first design two elementary models for interacting fermions that is free of randomness, whose solution in certain theoretical limit is a conformal field theory, and most importantly the fermion has a scaling dimension that depends on the charge density of the model. Then based on these elementary models we design two versions of lattice models which naturally give us $\varrho \sim T^{\alpha}$, and $\alpha \in[1,2)$ is tunable by charge density. Our models provide an alternative approach of studying various experimentally observed NFLs in a unified framework.

\section{THE ELEMENTARY MODELS}

We first give a brief review of the "tetrahedron" threeindex tensor model without any disorder, and in the large- $N$ limit their solutions mimic the better-known $\mathrm{SYK}_{4}$ model. As was discussed in Ref. 26, the original $\mathrm{U}\left(N_{a}\right) \times \mathrm{U}\left(N_{b}\right) \times \mathrm{O}\left(N_{c}\right)$ symmetric tetrahedron model can be written as

$$
H=\frac{g}{\sqrt{N_{a} N_{b} N_{c}}} \psi_{a_{1} b_{1} c_{1}}^{\dagger} \psi_{a_{2} b_{2} c_{1}}^{\dagger} \psi_{a_{1} b_{2} c_{2}} \psi_{a_{2} b_{1} c_{2}},
$$

where $a=1, \ldots, N_{a}, b=1, \ldots, N_{b}, c=1, \ldots, N_{c}$. One can prove that as long as

$$
0<\frac{N_{a}}{N_{b}}, \frac{N_{b}}{N_{c}}, \frac{N_{c}}{N_{a}}<\infty,
$$

this tensor model is dominated by the melonic diagrams in the large- $N_{a}, N_{b}, N_{c}$ limit (Fig. 1), and its solution is a conformal field theory fixed point in the infrared limit. At the conformal fixed point, the melonic diagrams can be summed by solving the Schwinger-Dyson equations which are identical to the original $\mathrm{SYK}_{4}$ model for the complex fermions 20 :

$$
\begin{aligned}
G\left(i \omega_{n}\right) & =\frac{1}{i \omega_{n}+\mu-\Sigma\left(i \omega_{n}\right)}, \\
\Sigma(\tau) & =-4 g^{2} G(\tau)^{2} G(-\tau),
\end{aligned}
$$

where the two-point Green's function $G(\tau)$ is defined as

$$
G(\tau) \delta_{a a^{\prime}} \delta_{b b^{\prime}} \delta_{c c^{\prime}}=-\left\langle\mathbb{T}_{\tau} \psi_{a b c}(\tau) \psi_{a^{\prime} b^{\prime} c^{\prime}}^{\dagger}(0)\right\rangle
$$

$\Sigma$ is the self energy, $\omega_{n}$ is fermionic Matsubara frequency $\omega_{n}=(2 n+1) \pi T, n \in \mathbb{Z}$, and $\tau$ is imaginary time. One key feature of this model is that in its conformal solution the fermions have the scaling dimension

$$
\Delta_{\psi}=\frac{1}{4}
$$

just like the $\mathrm{SYK}_{4}$ model.

This model certainly has many variants with the same large- $N$ solution. In Ref. 42 in order to make connection

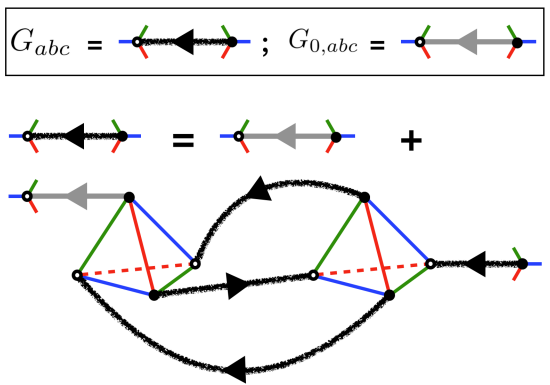

FIG. 1: The large- $N$ Schwinger-Dyson equation for various complex tetrahedron models.

to the cuprates, we constructed a lattice model based on a modified tensor model with the form

$$
H=\frac{g \mathcal{J}_{c_{1} c_{1}^{\prime}} \mathcal{J}_{c_{2} c_{2}^{\prime}}}{\sqrt{N_{a} N_{b} N_{c}}} \psi_{a_{1} b_{1} c_{1}}^{\dagger} \psi_{a_{2} b_{2} c_{1}^{\prime}}^{\dagger} \psi_{a_{1} b_{2} c_{2}} \psi_{a_{2} b_{1} c_{2}^{\prime}},
$$

where $\mathcal{J}$ is the antisymmetric matrix associated with the $\operatorname{Sp}\left(N_{c}\right)$ group and $\mathcal{J}_{c c^{\prime}} \psi_{c} \psi_{c^{\prime}}$ forms an $\operatorname{Sp}\left(N_{c}\right)$ singlet.

So far all the tetrahedron models are comprised of oneorbital of fermions with three indices and conformal dimension $1 / 4$ in the soluble limit. In this paper, we consider generalizations to two versions of "elementary" models each with two orbitals (types) of fermions $\psi$ and $\chi$, and a mutual four-fermion interaction. The existence of multi-orbitals of fermions is analogous to the situation in many heavy fermion systems, where most of the NFLs were observed. This simple generalization leads to some important new features: the conformal dimensions $\Delta_{\chi}$ and $\Delta_{\psi}$ can be tuned by changing the parameters, especially the particle density in the models. These elementary models enable us to build several lattice models for NFLs with different transport scalings with randomnessfree four-fermion interactions.

\section{A. Model A}

The first "elementary model" we construct takes the following form:

$$
\begin{aligned}
H_{0}^{A}= & \sum_{\substack{a_{1}, a_{2}, b_{1}, b_{2}=1 \\
N}}^{N} \sum_{c=1}^{M_{1}} \sum_{d=1}^{M_{2}} \frac{g}{N \sqrt{M}} \\
& \left(\psi_{a_{1}, b_{1}, c}^{\dagger} \psi_{a_{2}, b_{2}, c} \chi_{a_{1}, b_{2}, d}^{\dagger} \chi_{a_{2}, b_{1}, d}+\text { h.c. }\right),
\end{aligned}
$$

where $M=\sqrt{M_{1} M_{2}} . \psi$ and $\chi$ are two orbitals (types) of fermions each carries three indices. The model above is the simplest model with the desired features. It has continuous symmetries just like the original tetrahedron model, but these symmetries are not essential to our results. There are also some discrete symmetries that are more important for the solution, which will be spelled out later.

In the large- $N, M_{1}, M_{2}$ limit, just like the three-index tensor models, only the "melonic diagrams" dominate. 
The sum of all the melonic diagrams must satisfy the coupled Schwinger-Dyson (S-D) equations:

$$
\begin{aligned}
& G_{\psi}\left(i \omega_{n}\right)=\frac{1}{i \omega_{n}+\mu_{\psi}-\Sigma_{\psi}(i \omega)}, \\
& G_{\chi}\left(i \omega_{n}\right)=\frac{1}{i \omega_{n}+\mu_{\chi}-\Sigma_{\chi}(i \omega)},
\end{aligned}
$$

and the self energies are

$$
\begin{aligned}
& \Sigma_{\psi}^{A}(\tau)=-4 g^{2} \sqrt{\frac{M_{2}}{M_{1}}} G_{\psi}(\tau) G_{\chi}(\tau) G_{\chi}(-\tau), \\
& \Sigma_{\chi}^{A}(\tau)=-4 g^{2} \sqrt{\frac{M_{1}}{M_{2}}} G_{\chi}(\tau) G_{\psi}(\tau) G_{\psi}(-\tau),
\end{aligned}
$$

where we have introduced different chemical potentials $\mu_{\psi}, \mu_{\chi}$ for the two fermions to fix the particle densities.

Apparently, in this model the particle density of $\psi$ and $\chi$ are separately conserved, thus we can introduce filling factor $\mathcal{Q}_{\psi}, \mathcal{Q}_{\chi} \in(0,1)$ separately. $\mathcal{Q}_{\psi}$ is defined as

$$
\mathcal{Q}_{\psi}=\frac{\sum_{a, b, c}\left\langle\psi_{a, b, c}^{\dagger} \psi_{a, b, c}\right\rangle}{N^{2} M_{1}}
$$

and $\mathcal{Q}_{\chi}$ is defined accordingly. The role of the filling factors will be specified later and derived in detail in the supplementary material. With fixed filling factors $\mathcal{Q}_{\psi}$ and $\mathcal{Q}_{\chi}$, just like the original S-Y model Ref. 20, we should set $\Sigma\left(i \omega_{n}=0\right)=\mu$. Thus, we can redefine the self energy as

$$
\tilde{\Sigma}_{\psi / \chi}\left(i \omega_{n}\right)=\Sigma_{\psi / \chi}\left(i \omega_{n}\right)-\mu
$$

Now in the infrared limit, assuming the self-energy always dominates the $i \omega_{n}$ term in the infrared, the S-D equations are simplified as

$$
G_{\psi}\left(i \omega_{n}\right) \tilde{\Sigma}_{\psi}\left(i \omega_{n}\right)=G_{\chi}\left(i \omega_{n}\right) \tilde{\Sigma}_{\chi}\left(i \omega_{n}\right)=-1
$$

At general filling factors $\mathcal{Q}_{\psi}$ and $\mathcal{Q}_{\chi}$, and at zero temperature $T=0$, we use the following power law ansatz at complex frequency $z(\operatorname{Im}(z)>0,|z| \ll g)$ to solve the S-D equations

$$
\begin{aligned}
& G_{\psi}(z)=C_{\psi} \frac{e^{-i\left(\pi \Delta_{\psi}+\theta_{\psi}\right)}}{z^{1-2 \Delta_{\psi}}}, \\
& G_{\chi}(z)=C_{\chi} \frac{e^{-i\left(\pi \Delta_{\chi}+\theta_{\chi}\right)}}{z^{1-2 \Delta_{\chi}}},
\end{aligned}
$$

where the real parameters $C, \theta, \Delta$ satisfy

$$
\begin{array}{ll}
C_{\psi}>0, & -\pi \Delta_{\psi}<\theta_{\psi}<\pi \Delta_{\psi}, \\
C_{\chi}>0, & -\pi \Delta_{\chi}<\theta_{\chi}<\pi \Delta_{\chi} .
\end{array}
$$

There are in general six unknowns that we need to solve for: $C_{\psi / \chi}, \Delta_{\psi / \chi}$ and $\theta_{\psi / \chi}$. But through the S-D equations which are exact in the large- $N, M_{1}, M_{2}$ limit, we will be able to determine five of them: $C_{\psi}^{2} C_{\chi}^{2}, \Delta_{\psi / \chi}$ and $\theta_{\psi / \chi}$. The scaling dimensions $\Delta_{\psi / \chi}$ are the most important quantities which will determine the scaling of the transport coefficients, as we will calculate explicitly later. In the large- $N, M_{1}, M_{2}$ limit, only the product $C_{\psi}^{2} C_{\chi}^{2}$ is determined, while $C_{\psi}$ and $C_{\chi}$ may be determined separately through subleading diagrams.

The S-D equation, or the melonic diagrams, demand that the self energies at complex frequency $z, \operatorname{Im}(z)>0$ take the following form:

$$
\begin{aligned}
& \tilde{\Sigma}_{\psi}^{A}(z) \propto C_{\psi} C_{\chi}^{2} \sqrt{\frac{M_{2}}{M_{1}}} e^{i\left(\pi \Delta_{\psi}+\theta_{\psi}\right)} z^{1-2 \Delta_{\psi}}, \\
& \tilde{\Sigma}_{\chi}^{A}(z) \propto C_{\chi} C_{\psi}^{2} \sqrt{\frac{M_{1}}{M_{2}}} e^{i\left(\pi \Delta_{\chi}+\theta_{\chi}\right)} z^{1-2 \Delta_{\chi}} .
\end{aligned}
$$

Eventually the coupled S-D equations Eq. 15 lead to the following self-consistent equations:

$$
\begin{aligned}
& 2 g^{2} C_{\psi}^{2} C_{\chi}^{2} \sqrt{\frac{M_{2}}{M_{1}}} \frac{\cos \left(2 \pi \Delta_{\psi}\right)+\cos \left(2 \theta_{\chi}\right)}{\pi\left(1-2 \Delta_{\psi}\right) \sin \left(2 \pi \Delta_{\psi}\right)}=1, \\
& 2 g^{2} C_{\chi}^{2} C_{\psi}^{2} \sqrt{\frac{M_{1}}{M_{2}}} \frac{\cos \left(2 \pi \Delta_{\chi}\right)+\cos \left(2 \theta_{\psi}\right)}{\pi\left(1-2 \Delta_{\chi}\right) \sin \left(2 \pi \Delta_{\chi}\right)}=1 .
\end{aligned}
$$

The conformal dimensions $\Delta_{\psi}$ and $\Delta_{\chi}$ also must satisfy another relation, which physically guarantee that the system is at a fixed point controlled by the four fermion interaction:

$$
2 \Delta_{\psi}+2 \Delta_{\chi}=1
$$

Additionally, the filling factors $\mathcal{Q}_{\psi}$ and $\mathcal{Q}_{\chi}$ give further constraints on $\Delta_{\psi / \chi}$, and $\theta_{\psi / \chi}$ (please refer to the supplementary material):

$$
\begin{aligned}
& \mathcal{Q}_{\psi}=\frac{1}{2}-\frac{\theta_{\psi}}{\pi}-\left(\frac{1}{2}-\Delta_{\psi}\right) \frac{\sin \left(2 \theta_{\psi}\right)}{\sin \left(2 \pi \Delta_{\psi}\right)}, \\
& \mathcal{Q}_{\chi}=\frac{1}{2}-\frac{\theta_{\chi}}{\pi}-\left(\frac{1}{2}-\Delta_{\chi}\right) \frac{\sin \left(2 \theta_{\chi}\right)}{\sin \left(2 \pi \Delta_{\chi}\right)} .
\end{aligned}
$$

The five equations above, i.e. Eq. 22 to Eq. 26 involve five unknown real numbers that we need to solve for: $\Delta_{\psi}$, $\Delta_{\chi}, \theta_{\psi}, \theta_{\chi}$, and $C_{\psi}^{2} C_{\chi}^{2}$. These equations imply that the conformal dimension $\Delta_{\psi / \chi}$ can be tuned by the particle filling factors $\mathcal{Q}_{\psi}$ and $\mathcal{Q}_{\chi}$, as we will demonstrate explicitly later.

The imaginary time correlation function can be obtained by Fourier transforming Eq. 16 and Eq. 17 .

$$
\begin{array}{r}
G_{\psi / \chi}(\tau)=\frac{\mathcal{B}_{\psi / \chi}}{|\tau|^{2 \Delta_{\psi / \chi}}}, \quad \tau>0 \\
G_{\psi / \chi}(\tau)=-\frac{\mathcal{B}_{\psi / \chi}^{\prime}}{|\tau|^{2 \Delta_{\psi / \chi}}}, \quad \tau<0,
\end{array}
$$

Following the convention of the literatures on the complex SYK model (for example Ref. 22), we can introduce the spectral asymmetry $\mathcal{E}_{\psi / \chi}$

$$
e^{2 \pi \mathcal{E}_{\psi / \chi}}=\frac{\sin \left(\pi \Delta_{\psi / \chi}+\theta_{\psi / \chi}\right)}{\sin \left(\pi \Delta_{\psi / \chi}-\theta_{\psi / \chi}\right)}
$$


and the coefficient $\mathcal{B}_{\psi / \chi}, \mathcal{B}_{\psi / \chi}^{\prime}$ is related to $C_{\psi / \chi}$ as

$$
\begin{aligned}
\mathcal{B}_{\psi / \chi} & =-\frac{C_{\psi / \chi} \Gamma\left(2 \Delta_{\psi / \chi}\right) \sin \left(\pi \Delta_{\psi / \chi}+\theta_{\psi / \chi}\right)}{\pi} \\
\mathcal{B}_{\psi / \chi}^{\prime} & =-\frac{C_{\psi / \chi} \Gamma\left(2 \Delta_{\psi / \chi}\right) \sin \left(\pi \Delta_{\psi / \chi}-\theta_{\psi / \chi}\right)}{\pi} \\
& =\mathcal{B}_{\psi / \chi} e^{-2 \pi \mathcal{E}_{\psi / \chi}} .
\end{aligned}
$$

Although we cannot determine $C_{\psi}$ and $C_{\chi}$ separately from the $\mathrm{S}-\mathrm{D}$ equations, dimensional analysis determines that $\mathcal{B}_{\psi / \chi} \sim C_{\psi / \chi} \sim g^{-2 \Delta_{\psi / \chi}}$, thus $C_{\psi}^{2} C_{\chi}^{2} \sim 1 / g^{2}$.

The finite temperature solution can be obtained by performing the conformal mapping $\tau \rightarrow \frac{1}{\pi T} \tan (\pi T \tau)$, where $\tau$ becomes a periodic imaginary time coordinate with periodicity $1 / T$. Using the rules of reparametrization transformation, we obtain

$$
G(\tau)=\left\{\begin{array}{ll}
\mathcal{B} e^{-2 \pi \mathcal{E} T \tau}\left|\frac{\pi T}{\sin (\pi T \tau)}\right|^{2 \Delta} & 0<\tau<\frac{1}{T} \\
-\mathcal{B}^{\prime} e^{-2 \pi \mathcal{E} T \tau}\left|\frac{\pi T}{\sin (\pi T \tau)}\right|^{2 \Delta} & 0<-\tau<\frac{1}{T}
\end{array},\right.
$$

Now we are ready to solve the equations from Eq. 22 to Eq. 26. In general an analytic solution would be very tedious. But for the simplified case where $M_{1}=M_{2}$, there are only two parameters in this theory: $q_{\psi}=\mathcal{Q}_{\psi}-$ $1 / 2$ and $q_{\chi}=\mathcal{Q}_{\chi}-1 / 2$, and all the relevant quantities can be expanded as a polynomial of $q_{\psi}, q_{\chi}$. We also define $d=\Delta_{\psi}-1 / 4=1 / 4-\Delta_{\chi}$. Then Eq. 24 implies that $d_{\psi}=-d_{\chi}=d$. We will obtain analytic solutions for small $q_{\psi}$ and $q_{\chi}$.

In fact, in Eq. 26 and Eq. 25, we do not need to compute the exact prefactor before $\sin \left(2 \theta_{\psi}\right)$ and $\sin \left(2 \theta_{\chi}\right)$. Without loss of generality, we can assume the prefactor $f(\Delta, \theta)$ is a function of $\Delta$ and $\theta$, and some general constraints of the its form would be sufficient for the lowest nontrivial order of solutions as a polynomial of $q_{\psi / \chi}$. For example, $f(\Delta, \theta)$ must be consistent with the results in Ref. 50. When $q_{\psi}=q_{\chi}$, there is a $Z_{2}$ symmetry that exchanges $\psi$ and $\chi$, hence in this case $\Delta_{\psi}=\Delta_{\chi}=1 / 4$, or $d=d_{\psi}=-d_{\chi}=0$. And to be consistent with the result in Ref. 50, the $f(\Delta, \theta)$ function must satisfy

$$
f(1 / 4, \theta)=1 / 4,
$$

and this statement is independent of $\theta$. This is consistent with the result of Ref. 39 where it was found that $f(\Delta, \theta)$ does not depend on $\theta$ at all.

Under the particle-hole transformation, the Green's function $G(\tau)$ at filling factor $q_{\psi}, q_{\chi}$ will become $-G(-\tau)$ at filling factor $-q_{\psi},-q_{\chi}$. This implies that $d$ must be an even function of $q_{\psi}$ and $q_{\chi}$, while $\theta_{\psi}, \theta_{\chi}$ must be odd functions of $q_{\psi}, q_{\chi}$. If we assume $q_{\psi} \sim q_{\chi} \sim q \ll 1$, to the lowest order expansion of $q_{\psi}$ and $q_{\chi}, d \sim\left(q_{\psi}^{2}-q_{\chi}^{2}\right)$, which follows from the aforementioned fact that $d=0$ when $q_{\psi}=q_{\chi}$. Thus to the lowest nontrivial order of expansion of $q$, we can just take $f(\Delta, \theta)=1 / 4+O\left(q_{\psi}^{2}-q_{\chi}^{2}\right)+O\left(q^{3}\right)$.

All the five equations from Eq. 22 to Eq. 26 can be expanded as a polynomial of $q_{\psi}$ and $q_{\chi}$. And at the lowest nontrivial order, we obtain the following analytic solutions:

$$
\begin{aligned}
\theta_{\psi} & =-\frac{2 \pi q_{\psi}}{\pi+2}+O\left(q^{3}\right) \\
\theta_{\chi} & =-\frac{2 \pi q_{\chi}}{\pi+2}+O\left(q^{3}\right) \\
\Delta_{\psi} & =\frac{1}{4}+d=\frac{1}{4}+\frac{2 \pi^{2}\left(q_{\psi}^{2}-q_{\chi}^{2}\right)}{(\pi+2)^{2}(\pi-2)}+O\left(q^{4}\right) \\
\Delta_{\chi} & =\frac{1}{4}-d=\frac{1}{4}-\frac{2 \pi^{2}\left(q_{\psi}^{2}-q_{\chi}^{2}\right)}{(\pi+2)^{2}(\pi-2)}+O\left(q^{4}\right)
\end{aligned}
$$

These solutions are consistent with all the previous observations, and also consistent with numerical solutions of the equations

\section{B. Model B}

Another elementary model that we will start with is also constructed with two orbitals of fermions, each with three indices. The Hamiltonian takes the following form:

$$
\begin{aligned}
H_{0}^{B} & =\sum_{\substack{a_{1}, a_{2}, b_{1}, b_{2}=1 \\
\left(\psi_{c, c^{\prime}=1}^{\dagger}\right.}}^{N} \sum_{d, d^{\prime}=1}^{M_{1}} \frac{g}{N \sqrt{M}} \mathcal{J}_{c, c^{\prime}}^{\psi} \mathcal{J}_{d, d^{\prime}}^{\chi} \\
& \left.\psi_{a_{1}, b_{1}, c} \psi_{a_{2}, b_{2}, c^{\prime}}^{\dagger} \chi_{a_{1}, b_{2}, d} \chi_{a_{2}, b_{1}, d^{\prime}}+\text { h.c. }\right)
\end{aligned}
$$

Here $\psi_{c}$ and $\chi_{d}$ form fundamental representation of $\operatorname{Sp}\left(M_{1}\right)$ and $\operatorname{Sp}\left(M_{2}\right)$ group. $\mathcal{J}_{c, c^{\prime}}^{\psi} \psi_{c} \psi_{c^{\prime}}$ and $\mathcal{J}_{d, d^{\prime}}^{\chi} \chi_{d} \chi_{d^{\prime}}$ form singlets under $\operatorname{Sp}\left(M_{1}\right)$ and $\operatorname{Sp}\left(M_{2}\right)$ respectively.

Although both model $\mathrm{A}$ and model $\mathrm{B}$ share a similar three-index structure, there are some fundamental differences between them. First of all, the particle density of $\psi$ and $\chi$ are no longer separately conserved in model B. Only the total particle density is conserved. Thus, we should introduce

$$
\mathcal{Q}=\frac{M_{1} \mathcal{Q}_{\psi}+M_{2} \mathcal{Q}_{\chi}}{M_{1}+M_{2}} \in(0,1)
$$

as a "total" filling factor, Notice that $Q_{\psi}$ and $Q_{\chi}$ are defined as the expectation values of $\psi$ and $\chi$ fermion number operator (Eq. 13), while only $\mathcal{Q}$ is a conserved quantity in this case.

Secondly and very importantly, the self energies are different compared with those of model A, based on the melonic diagrams:

$$
\begin{aligned}
& \Sigma_{\psi}^{B}(\tau)=-4 g^{2} \sqrt{\frac{M_{2}}{M_{1}}} G_{\chi}(\tau)^{2} G_{\psi}(-\tau), \\
& \Sigma_{\chi}^{B}(\tau)=-4 g^{2} \sqrt{\frac{M_{1}}{M_{2}}} G_{\psi}(\tau)^{2} G_{\chi}(-\tau),
\end{aligned}
$$

Again, we want to solve the coupled S-D equations Eq. 15 self-consistently in the conformal limit, and we still use the power law ansatz Eq. 16 and Eq. 17. We found that the self energies $\tilde{\Sigma}_{\psi}^{B}, \tilde{\Sigma}_{\chi}^{B}$ can still be written as 
the form of Eq. 20. Eq. 21. But now the self-consistency of the S-D equation imposes another constraint on $\theta_{\psi}, \theta_{\chi}$ (for more details, please refer to the supplementary material appendix A):

$$
\frac{\sin \left(\pi \Delta_{\psi}+\theta_{\psi}\right)}{\sin \left(\pi \Delta_{\psi}-\theta_{\psi}\right)}=\frac{\sin \left(\pi \Delta_{\chi}+\theta_{\chi}\right)}{\sin \left(\pi \Delta_{\chi}-\theta_{\chi}\right)}
$$

which implies that the two types of fermions have the same spectral asymmetry. Under this constraint, the SD equation Eq.15leads to the same expressions as Eq. 22 and Eq. 23.

In addition, we have verified in the supplementary material that the expectation values of the particle numbers for $\psi$ and $\chi$ fermions share the same expressions Eq. 25 and Eq. 26 as model A. The total filling factor $\mathcal{Q}$ imposes further constraints on $\Delta_{\psi / \chi}$, and $\theta_{\psi / \chi}$

$$
\begin{aligned}
\mathcal{Q} & =\frac{1}{2}-\frac{M_{1} \theta_{\psi}+M_{2} \theta_{\chi}}{\pi\left(M_{1}+M_{2}\right)} \\
& -\frac{M_{1} \Delta_{\chi} \sin \left(2 \theta_{\psi}\right)+M_{2} \Delta_{\psi} \sin \left(2 \theta_{\chi}\right)}{\sin \left(2 \pi \Delta_{\psi / \chi}\right)\left(M_{1}+M_{2}\right)},
\end{aligned}
$$

where $\Delta_{\psi / \chi}$ can be either $\Delta_{\psi}$ or $\Delta_{\chi}$ due to Eq. 24 .

Still, we have five equations that involve five unknown real quantities $\Delta_{\psi}, \Delta_{\chi}, \theta_{\psi}, \theta_{\chi}$, and $C_{\psi}^{2} C_{\chi}^{2}$. Compared to model A, the conditions that $\mathcal{Q}_{\psi}$ and $\mathcal{Q}_{\chi}$ are fixed separately is replaced by fixing $\mathcal{Q}$, together with the constraint Eq. 37. Now the conformal dimension $\Delta_{\psi / \chi}$ can be tuned by changing the total particle filling factor $\mathcal{Q}$.

\section{LATTICE MODELS FOR NFLS}

\section{A. Lattice model (1)}

Based on the elementary models constructed in the previous section, we can construct lattice models with the desired resistivity scaling $\varrho \sim T^{\alpha}$ with $\alpha \in[1,2)$. Our first lattice model is constructed with coupled clusters (following the previous efforts 38 , 41 of constructing the strange metal phase with the $\mathrm{SYK}_{4}$-like clusters), and the physics on each cluster $\boldsymbol{r}$ is described by Eq. 8 or Eq. 33. which is the leading energy scale of the system. Different clusters are coupled together through hoppings of both $\psi$ and $\chi$ :

$$
H=\sum_{\boldsymbol{r}} H_{0}^{A / B}(\boldsymbol{r})-\sum_{\left\langle\boldsymbol{r}, \boldsymbol{r}^{\prime}\right\rangle}\left(t_{1} \psi_{\boldsymbol{r}}^{\dagger} \psi_{\boldsymbol{r}^{\prime}}+t_{2} \chi_{\boldsymbol{r}}^{\dagger} \chi_{\boldsymbol{r}^{\prime}}\right)+\ldots
$$

The indices of $\psi$ and $\chi$ are summed over in the equation above. Although the $t$-terms are expected to drive the system into a Fermi liquid state at low energy, our goal is to construct a NFL phase at a finite energy/temperature window, which is where most of the NFLs are observed experimentally. Thus let us focus on the finite energy window where $H_{0}^{A / B}$ is dominant, and the hopping term is perturbative. (a)

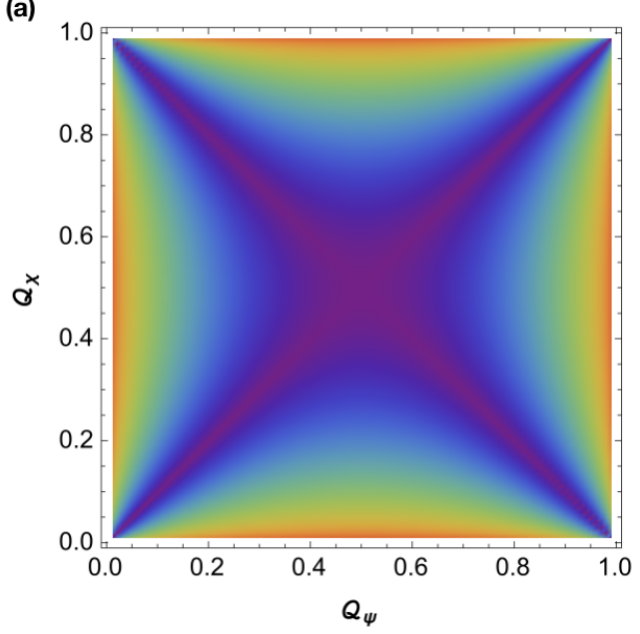

(b)

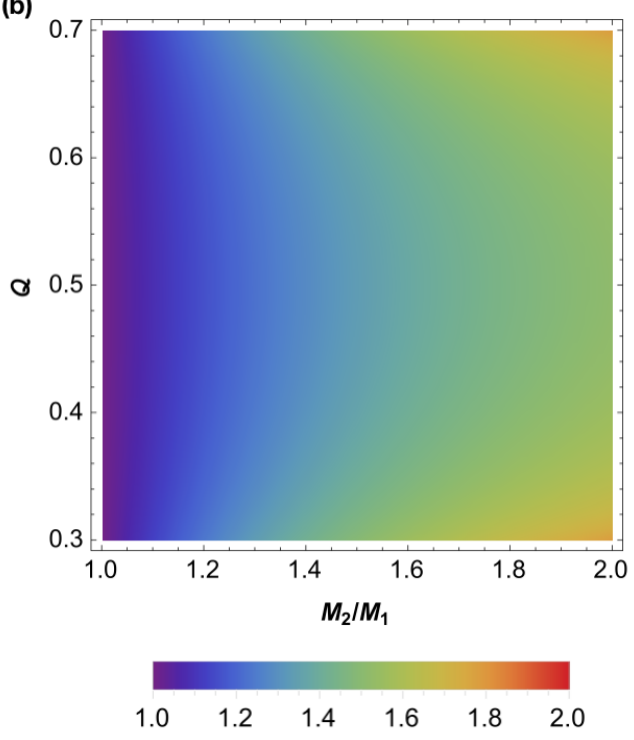

FIG. 2: The relation between the transport scaling power $\alpha$ (defined as resistivity $\varrho \sim T^{\alpha}$ ) and parameters in the lattice models for NFLs. (a) $\alpha$ plotted against $\mathcal{Q}_{\psi}$ and $\mathcal{Q}_{\chi}$ with $M_{2} / M_{1}=1$ for the lattice model (1) with the on-cluster Hamiltonian $H_{0}^{A}(\boldsymbol{r}) ;(b) \alpha$ plotted against $\mathcal{Q}$ and $M_{2} / M_{1}$, for lattice model (1) with the on-cluster Hamiltonian $H_{0}^{B}(\boldsymbol{r})$; and also the lattice model (2) Eq. 49

The electric current operator of model Eq. 39 can be obtained by coupling the model to the external electromagnetic field, and perform functional derivative of the external field:

$$
J_{\boldsymbol{\delta}}=\sum_{\boldsymbol{r}} i t_{1} \psi_{\boldsymbol{r}}^{\dagger} \psi_{\boldsymbol{r}+\boldsymbol{\delta}}+i t_{2} \chi_{\boldsymbol{r}}^{\dagger} \chi_{\boldsymbol{r}+\boldsymbol{\delta}}+\text { H.c. }
$$

In order to compute the electric conductivity, we define the imaginary-time current-current correlation function as $C(J, J ; \tau)=\left\langle\mathbb{T}_{\tau} J(\tau) J(0)\right\rangle$. The leading order nonzero contribution takes the form

$$
\frac{C(J, J ; \tau)}{\mathcal{N}}=-2 t_{1}^{2} G_{\psi}(\tau) G_{\psi}(-\tau)-2 t_{2}^{2} G_{\chi}(\tau) G_{\chi}(-\tau),
$$


where $\mathcal{N}$ is $\mathcal{N}=N^{2} M V$ with $V$ being the size of the lattice.

Then we perform Fourier transformation of $C(J, J ; \tau)$ to obtain correlation function in the Matsubara frequency space:

$$
\frac{C\left(J, J ; i \omega_{n}\right)}{\mathcal{N}}=\frac{C_{\psi}\left(J, J ; i \omega_{n}\right)}{\mathcal{N}}+\frac{C_{\chi}\left(J, J ; i \omega_{n}\right)}{\mathcal{N}},
$$

where $C_{\psi}$ is calculated as

$$
\frac{C_{\psi}\left(J, J ; i \omega_{n}\right)}{\mathcal{N}}=2 t_{1}^{2} \int_{0}^{\frac{1}{T}} d \tau e^{i \omega_{n} \tau} G_{\psi}(\tau) G_{\psi}\left(\frac{1}{T}-\tau\right)
$$

which is exact in the large- $N, M_{1}, M_{2}$ limit, and $C_{\chi}$ has a similar expression.

When $0<\Delta_{\psi}<1 / 4$, the integral Eq. 43 has a finite expression, but it diverges when $1 / 4 \leq \Delta_{\psi}<1 / 2$. For
$1 / 4 \leq \Delta_{\psi}<1 / 2$, we regulate the integral by introducing a small positive cutoff $\delta>0$ :

$$
\int_{0}^{\frac{1}{T}} \rightarrow \int_{\delta}^{\frac{1}{T}-\delta}
$$

There is a $\mathcal{O}(\log \delta)$ divergence when $\Delta_{\psi}=1 / 4$, and a $\mathcal{O}\left(1 / \delta^{4 \Delta-1}\right)$ divergence when $1 / 4<\Delta_{\psi}<1 / 2$. The divergence is in the real part but not the imaginary part of the correlation function, hence does not contribute to the conductivity, thus the divergence can be removed in order to calculate the conductivity. The retarded/advanced correlation function $C^{R / A}(J, J ; \omega)$ can then be derived by taking $z \rightarrow \omega \pm i 0^{+}$. And eventually using the relation $\sigma(\omega)=\frac{1}{i \omega} C^{R}(J, J ; \omega)$, we find the real part of the optical conductivity

$$
\operatorname{Re}[\sigma(\omega)] \sim \frac{t_{1}^{2} \mathcal{B}_{\psi}^{2} e^{-2 \pi \mathcal{E}_{\psi}}}{T^{2-4 \Delta_{\psi}}} \Upsilon\left(\Delta_{\psi}, \frac{\omega}{T}\right)+\frac{t_{2}^{2} \mathcal{B}_{\chi}^{2} e^{-2 \pi \mathcal{E}_{\chi}}}{T^{2-4 \Delta_{\chi}}} \Upsilon\left(\Delta_{\chi}, \frac{\omega}{T}\right),
$$

where we have introduced the scaling function

$$
\Upsilon\left(\Delta, \frac{\omega}{T}\right)=\frac{(2 \pi)^{4 \Delta-1}}{\Gamma(4 \Delta) \cos (2 \pi \Delta)} \frac{2 \pi T}{\omega} \operatorname{Im}\left[\frac{\Gamma\left(2 \Delta+\frac{\omega}{i 2 \pi T}\right)}{\Gamma\left(1-2 \Delta+\frac{\omega}{i 2 \pi T}\right)}\right] \quad 0<\Delta<1 / 2 .
$$

One can check that when $\Delta=1 / 4$, the scaling function above reproduces the scaling function for $\mathrm{SYK}_{4}$-like models 42

$$
\Upsilon(\Delta=1 / 4, \omega / T)=\frac{\pi \tanh (\omega / 2 T)}{\omega / 2 T} .
$$

The DC limit $\omega \rightarrow 0$ of the scaling function $\Upsilon(\Delta, 0)$ is a function of $\Delta$ which takes finite positive values for $\Delta \in(0,1 / 2)$. Since $2 \Delta_{\psi}+2 \Delta_{\chi}=1$, the final result of the DC conductivity takes the following form

$$
\operatorname{Re}[\sigma] \sim \frac{A}{T^{2-4 \Delta}}+\frac{B}{T^{4 \Delta}}
$$

where $\Delta$ takes values in $0<\Delta<1 / 2$. The constants $A \sim t_{1}^{2} \mathcal{B}_{\psi}^{2} \sim t_{1}^{2} / g^{4 \Delta}$, and $B \sim t_{2}^{2} \mathcal{B}_{\chi}^{2} \sim t_{2}^{2} / g^{2-4 \Delta}$. Hence when $T<g$, the $A / T^{2-4 \Delta}$ part of the DC conductivity will dominate for $0<\Delta<1 / 4$, and $B / T^{4 \Delta}$ dominates for $1 / 4<\Delta<1 / 2$. Thus, in a finite temperature window for $T$ lower than the dominant energy scale $g$, and higher than the infrared scale below which the hopping terms become nonperturbative, we are able to realize non-fermi liquid behaviors with resistivity $\varrho \sim T^{\alpha}$, and $\alpha \in[1,2)$ depends on parameters in the theory, especially the filling factors in the model.
The relation between $\alpha$ and the filling factors is plotted in Fig. 2, If we start with model A on every cluster, $\alpha$ will depend on both $Q_{\chi}$ and $Q_{\psi}$ even when $M_{1}=M_{2}$; if we start with model $\mathrm{B}$, then $\alpha$ depends on the total filling factor $Q$ when $M_{1} \neq M_{2}$.

\section{B. Lattice model (2)}

In this section we we propose another different construction of lattice model for NFL, by relating two of the three tensor indices to the lattice site coordinates of a two dimensional square lattice.

The dominant interaction in this model is

$$
\begin{gathered}
H=\sum_{j} \sum_{r, r^{\prime}=-(N-1) / 2}^{(N-1) / 2} \sum_{c, c^{\prime}=1}^{M_{1}} \sum_{d, d^{\prime}=1}^{M_{2}} \frac{g}{N \sqrt{M}} \mathcal{J}_{c, c^{\prime}}^{\psi} \mathcal{J}_{d, d^{\prime}}^{\chi} \\
\left(\psi_{j_{x}, j_{y}, c}^{\dagger} \psi_{j_{x}+r, j_{y}+r^{\prime}, c^{\prime}}^{\dagger} \chi_{j_{x}, j_{y}+r^{\prime}, d} \chi_{j_{x}+r, j_{y}, d^{\prime}}+\text { h.c. }\right) .
\end{gathered}
$$

This Hamiltonian is motivated by and resembles $H_{0}^{B}$. $\left(j_{x}, j_{y}\right)$ represents the $x$ and $y$ coordinates of the lattice site $j$. Physically $\psi_{c}$ and $\chi_{d}$ can be thought of as two types of fermions with $M_{1}=2 J_{1}+1$ and $M_{2}=2 J_{2}+1$ total angular momentum components, and the Hamiltonian represents the process of tunnelling between the pair 
singlets of $\chi$ and $\psi$. The cluster model in the previous section is insensitive to the spatial dimensions, while the construction of Eq. 49 most naturally applies to a two dimensional system.

In Eq. 49, we always take the thermodynamics limit first (the sum of $j$ is taken on a square lattice with infinite size). Then in the large- $N$ (in this model larger $-N$ means longer range interaction) and large- $M_{1}, M_{2}$ limit, the fermion Green's function is still dominated by the "melonic diagrams" and hence the Schwinger-Dyson equations, and their solutions, remain the same as model $H_{0}^{B}$. Notice that the single fermion Green's function is completely local in space, which is guaranteed by the fact that the Eq. 49 conserves the center of mass.

In addition to the dominant interaction, we will also turn on a single-particle hopping term as perturbations. Because Eq. 49 conserves the center of mass of the electrons, the interaction Eq. 49 alone cannot transport electric charge. Thus the electric current operator only comes from the electron hopping terms. In the soluble large$\left(N, M_{1}, M_{2}\right)$ limit, we formally generalize the electric current operator to the following form

$$
\begin{aligned}
J_{x} & =\frac{i t_{1}}{\sqrt{N M_{1}}}\left(\sum_{c} \psi_{j, c}^{\dagger} \psi_{j+\hat{x}, c}+\sqrt{\frac{N-1}{2}} \psi_{j, c}^{\dagger} \psi_{j+\hat{x} \pm \hat{y}, c}\right) \\
& +\frac{i t_{2}}{\sqrt{N M_{2}}}\left(\sum_{d} \chi_{j, d}^{\dagger} \chi_{j+\hat{x}, d}+\sqrt{\frac{N-1}{2}} \chi_{j, d}^{\dagger} \chi_{j+\hat{x} \pm \hat{y}, d}\right) \\
& + \text { H.c. }
\end{aligned}
$$

This electric current density can be derived by designing a corresponding single-electron hopping term in the large- $\left(N, M_{1}, M_{2}\right)$ limit (which involves both nearestand second-neighbor hopping) and coupling it to the external electromagnetic field.

Using the large- $\left(N, M_{1}, M_{2}\right)$ solution of Eq. 49 , we can repeat all the calculations for conductivity as we did for the previous model (1), and we arrive at the same expression of conductivity Eq. 45. Thus, we again have tunability of transport scalings within this construction. The exponent $\alpha$ of $\varrho \sim T^{\alpha}$ is plotted against the filling factor $Q$ and $M_{2} / M_{1}$ in Fig. $2 p$.

\section{SUMMARY AND DISCUSSION}

We constructed two examples of lattice models for nonfermi liquid states whose DC resistivity scalings are tun- able by adjusting the charge density, which is a phenomenon observed in many physical systems. Our lattice models are based on two versions of "elementary" models with randomness free four fermion interactions, which are soluble in certain theoretical limit just like the SYK model and the fermion tensor models. But unlike the previous models, our elementary models have tunable fermion scaling dimensions in their conformal solutions.

In this work we assumed that both orbitals (types) of the fermions in the model carry electric charges. But at least for model $\mathrm{A}$, where the number of each type of fermions is conserved separately, we can also assume that one of the two types of fermions are charge neutral slave particles, which comes from "fractionalizing" the localized spins. This perspective is similar to the the case in the original Sachdev-Ye model 20 , and also similar to a series of recent studies 3941 . In this case, the slave fermions will be coupled to a U(1) gauge field, whose effect in the large $-N$ limit is expected to be suppressed, and the solution of our model in the large $-N$ limit remains unchanged. In this case the electric transport only comes from one of the two orbitals of the fermions, and it is still tunable by changing the charge density of the system.

In Ref. 2842 , it was shown that the SYK-type of models are instable against extra marginally relevant fourfermion interactions, and these perturbations can lead to instability at low energy/temperature. In experiment, many of the observed NFLs are preempted by ordered phases (for example superconductivity) at low temperature. Also, it was shown in Ref. 50 that the $1 / N$ effect of the original Sachdev-Ye model plays a role only at an exponentially suppressed energy scale, and at finite temperature there is a wide window where the conformal solution of the Sachdev-Ye model applies. Similar effects were shown for the SYK model and also the three-index tensor models by studying the subleading order of the Feynmann diagrams 51. All these analysis can be performed for our models as well, which we will defer to future study.

The authors thank Yingfei Gu for very helpful discussions. Chao-Ming Jian's research at KITP is supported by the Gordon and Betty Moore Foundations EPiQS Initiative through Grant GBMF4304. Cenke $\mathrm{Xu}$ is supported by the David and Lucile Packard Foundation. 


\section{Appendix A: More details about Self energies}

a. Model $A$ Using the S-D equations, the fermion self-energies $\Sigma_{\psi / \chi}^{A}$ in imaginary time reads

$$
\begin{aligned}
& \tilde{\Sigma}_{\psi}^{A}(\tau)=-2 g^{2} C_{\psi} C_{\chi}^{2} \sqrt{\frac{M_{2}}{M_{1}}} \frac{\left(\cos \left(2 \pi \Delta_{\psi}\right)+\cos \left(2 \theta_{\chi}\right)\right) \Gamma\left(1-2 \Delta_{\psi}\right) \sin \left(\pi \Delta_{\psi}+\operatorname{sgn}(\tau) \theta_{\psi}\right)}{\pi^{2} \sin \left(2 \pi \Delta_{\psi}\right)} \frac{\operatorname{sgn}(\tau)}{|\tau|^{2-2 \Delta_{\psi}},} \\
& \tilde{\Sigma}_{\chi}^{A}(\tau)=-2 g^{2} C_{\chi} C_{\psi}^{2} \sqrt{\frac{M_{1}}{M_{2}}} \frac{\left(\cos \left(2 \pi \Delta_{\chi}\right)+\cos \left(2 \theta_{\psi}\right)\right) \Gamma\left(1-2 \Delta_{\chi}\right) \sin \left(\pi \Delta_{\chi}+\operatorname{sgn}(\tau) \theta_{\chi}\right)}{\pi^{2} \sin \left(2 \pi \Delta_{\chi}\right)} \frac{\operatorname{sgn}(\tau)}{|\tau|^{2-2 \Delta_{\chi}}} .
\end{aligned}
$$

After Fourier transformation, the self-energy at complex frequency $z, \operatorname{Im}(z)>0$ reads

$$
\begin{aligned}
& \tilde{\Sigma}_{\psi}^{A}(z)=-2 g^{2} C_{\psi} C_{\chi}^{2} \sqrt{\frac{M_{2}}{M_{1}}} \frac{\cos \left(2 \pi \Delta_{\psi}\right)+\cos \left(2 \theta_{\chi}\right)}{\pi\left(1-2 \Delta_{\psi}\right) \sin \left(2 \pi \Delta_{\psi}\right)} e^{i\left(\pi \Delta_{\psi}+\theta_{\psi}\right)} z^{1-2 \Delta_{\psi}}, \\
& \tilde{\Sigma}_{\chi}^{A}(z)=-2 g^{2} C_{\chi} C_{\psi}^{2} \sqrt{\frac{M_{1}}{M_{2}}} \frac{\cos \left(2 \pi \Delta_{\chi}\right)+\cos \left(2 \theta_{\psi}\right)}{\pi\left(1-2 \Delta_{\chi}\right) \sin \left(2 \pi \Delta_{\chi}\right)} e^{i\left(\pi \Delta_{\chi}+\theta_{\chi}\right)} z^{1-2 \Delta_{\chi}} .
\end{aligned}
$$

We can see that the self-energy for model A automatically takes the form $\Sigma^{A}(z) \propto e^{i(\pi \Delta+\theta)} z^{1-2 \Delta}$ with a real factor.

b. Model B We then consider model B. Using the S-D equations, the self-energies $\Sigma_{\psi / \chi}^{B}$ in imaginary time are

$$
\begin{aligned}
& \tilde{\Sigma}_{\psi}^{B}(\tau)=-4 g^{2} C_{\psi} C_{\chi}^{2} \sqrt{\frac{M_{2}}{M_{1}}} \frac{\cos ^{2}\left(\pi \Delta_{\psi}-\operatorname{sgn}(\tau) \theta_{\chi}\right) \sin \left(\pi \Delta_{\psi}-\operatorname{sgn}(\tau) \theta_{\psi}\right) \Gamma\left(1-2 \Delta_{\psi}\right)}{\pi^{2} \sin \left(2 \pi \Delta_{\psi}\right)} \frac{\operatorname{sgn}(\tau)}{|\tau|^{2-2 \Delta_{\psi}},} \\
& \tilde{\Sigma}_{\chi}^{B}(\tau)=-4 g^{2} C_{\chi} C_{\psi}^{2} \sqrt{\frac{M_{1}}{M_{2}}} \frac{\cos ^{2}\left(\pi \Delta_{\chi}-\operatorname{sgn}(\tau) \theta_{\psi}\right) \sin \left(\pi \Delta_{\chi}-\operatorname{sgn}(\tau) \theta_{\chi}\right) \Gamma\left(1-2 \Delta_{\chi}\right)}{\pi^{2} \sin \left(2 \pi \Delta_{\chi}\right)} \frac{\operatorname{sgn}(\tau)}{|\tau|^{2-2 \Delta_{\chi}}} .
\end{aligned}
$$

Again, after Fourier transformation, the self-energy with imaginary frequency reads:

$\tilde{\Sigma}_{\psi}^{B}(z)=-g^{2} C_{\psi} C_{\chi}^{2} \sqrt{\frac{M_{2}}{M_{1}}} \frac{e^{-i 2\left(\pi \Delta_{\psi}+\theta_{\chi}+\theta_{\psi}\right)}\left(\left(-1+e^{4 i \theta_{\chi}}\right) e^{2 i\left(\pi \Delta_{\psi}+\theta_{\psi}\right)}+2 e^{2 i\left(\pi \Delta_{\psi}+\theta_{\chi}\right)}+e^{4 i \pi \Delta_{\psi}}+1\right)}{\pi\left(1-2 \Delta_{\psi}\right) \sin \left(2 \pi \Delta_{\psi}\right)} e^{i\left(\pi \Delta_{\psi}+\theta_{\psi}\right)} z^{1-2 \Delta_{\psi}}$,

$\tilde{\Sigma}_{\chi}^{B}(z)=-g^{2} C_{\chi} C_{\psi}^{2} \sqrt{\frac{M_{1}}{M_{2}}} \frac{e^{-i 2\left(\pi \Delta_{\chi}+\theta_{\chi}+\theta_{\psi}\right)}\left(\left(-1+e^{4 i \theta_{\psi}}\right) e^{2 i\left(\pi \Delta_{\chi}+\theta_{\chi}\right)}+2 e^{2 i\left(\pi \Delta_{\chi}+\theta_{\psi}\right)}+e^{4 i \pi \Delta_{\chi}}+1\right)}{\pi\left(1-2 \Delta_{\chi}\right) \sin \left(2 \pi \Delta_{\chi}\right)} e^{i\left(\pi \Delta_{\chi}+\theta_{\chi}\right)} z^{1-2 \Delta_{\chi}}$.

The self-consistency of the S-D equation demands the self-energy take the form $\Sigma^{B}(z)=-C^{-1} e^{i(\pi \Delta+\theta)} z^{1-2 \Delta}$ with a real pre-factor $C$. Demanding the imaginary part of $C$ vanish leads to

$$
\begin{aligned}
& \cos \left(\theta_{\chi}+\theta_{\psi}\right)\left(\sin ^{2}\left(\pi \Delta_{\psi}\right) \sin \left(\theta_{\chi}\right) \cos \left(\theta_{\psi}\right)-\cos ^{2}\left(\pi \Delta_{\psi}\right) \cos \left(\theta_{\chi}\right) \sin \left(\theta_{\psi}\right)\right)=0, \\
& \cos \left(\theta_{\chi}+\theta_{\psi}\right)\left(\sin ^{2}\left(\pi \Delta_{\chi}\right) \sin \left(\theta_{\psi}\right) \cos \left(\theta_{\chi}\right)-\cos ^{2}\left(\pi \Delta_{\chi}\right) \cos \left(\theta_{\psi}\right) \sin \left(\theta_{\chi}\right)\right)=0 .
\end{aligned}
$$

These equations can be simplified as

$$
\frac{\tan \left(\theta_{\psi}\right)}{\tan \left(\pi \Delta_{\psi}\right)}=\frac{\tan \left(\theta_{\chi}\right)}{\tan \left(\pi \Delta_{\chi}\right)}
$$

where we have used $\Delta_{\psi}+\Delta_{\chi}=1 / 2$ to simplify the equations. In fact, we can rewrite Eq. A11 as

$$
\frac{\sin \left(\pi \Delta_{\psi}+\theta_{\psi}\right)}{\sin \left(\pi \Delta_{\psi}-\theta_{\psi}\right)}=\frac{\sin \left(\pi \Delta_{\chi}+\theta_{\chi}\right)}{\sin \left(\pi \Delta_{\chi}-\theta_{\chi}\right)}
$$

which implies that the two types of fermions have the same spectral asymmetry.

The S-D equation also requires

$$
\begin{aligned}
C_{\psi}^{-2} C_{\chi}^{-2} & =2 g^{2} \sqrt{\frac{M_{2}}{M_{1}}} \frac{\cos \left(2 \pi \Delta_{\psi}\right) \cos \left(2\left(\theta_{\chi}+\theta_{\psi}\right)\right)+\cos \left(2 \theta_{\psi}\right)}{\pi\left(1-2 \Delta_{\psi}\right) \sin \left(2 \pi \Delta_{\psi}\right)}, \\
C_{\chi}^{-2} C_{\psi}^{-2} & =2 g^{2} \sqrt{\frac{M_{1}}{M_{2}}} \frac{\cos \left(2 \pi \Delta_{\chi}\right) \cos \left(2\left(\theta_{\chi}+\theta_{\psi}\right)\right)+\cos \left(2 \theta_{\chi}\right)}{\pi\left(1-2 \Delta_{\chi}\right) \sin \left(2 \pi \Delta_{\chi}\right)} .
\end{aligned}
$$

Imposing the constraints Eq. 37 or Eq. A12, we recover exactly the same self-consistent equations Eq. 22 and Eq. 23 as the model A. 


\section{Appendix B: Luttinger-Ward calculation}

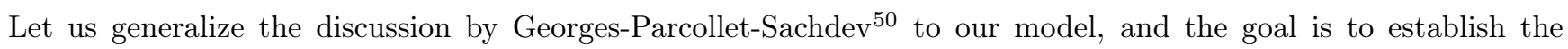
relation between the filling factors (particle density) $\mathcal{Q}_{\psi}, \mathcal{Q}_{\chi}$ of model $A$, and $\mathcal{Q}$ of model $B$ to the most relevant quantities such as $\Delta_{\psi / \chi}$ and $\theta_{\psi / \chi}$.

In the real-time formalism, at zero temperature, the filling factor can be evaluated by computing the following integral $\sqrt{50}$

$$
i \mathbb{P} \int_{-\infty}^{+\infty} \frac{d \omega}{2 \pi} e^{i \omega 0^{+}}\left(\partial_{\omega} \log G(\omega)-G(\omega) \partial_{\omega} \tilde{\Sigma}(\omega)\right)
$$

where $G(\omega)=G^{R}(\omega) \Theta(\omega)+G^{A}(\omega) \Theta(-\omega)$ is the time-ordered Green function with $\Theta(\omega)$ being the Heaviside step function, and $G^{R / A}(\omega)=G\left(\omega \pm i 0^{+}\right)$is the real-time retarded/advanced Green's function obtained by replacing $i \omega_{n}$ by $\omega \pm i 0^{+}$in the imaginary-time Green's function. We use $\mathbb{P}$ to denote the the principal value of the integral $\mathbb{P} \int_{-\infty}^{+\infty}=\int_{-\infty}^{-\delta}+\int_{+\delta}^{+\infty}$ with a small positive cut off $\delta>\sqrt{50}$.

Through the same line of arguments in Appendix A of Ref. 50 (also see Appendix D of Ref. 39), the filling factors for both fermions $\psi$ and $\chi$ are

$$
\begin{aligned}
& \mathcal{Q}_{\psi}=\frac{1}{2}-\frac{\theta_{\psi}}{\pi}-i \mathbb{P} \int_{-\infty}^{+\infty} \frac{d \omega}{2 \pi} e^{i \omega 0^{+}} G_{\psi}(\omega) \partial_{\omega} \tilde{\Sigma}_{\psi}(\omega), \\
& \mathcal{Q}_{\chi}=\frac{1}{2}-\frac{\theta_{\chi}}{\pi}-i \mathbb{P} \int_{-\infty}^{+\infty} \frac{d \omega}{2 \pi} e^{i \omega 0^{+}} G_{\chi}(\omega) \partial_{\omega} \tilde{\Sigma}_{\chi}(\omega) .
\end{aligned}
$$

We are going to calculate the integral

$$
\mathcal{I}_{\psi / \chi}^{A / B}=i \mathbb{P} \int_{-\infty}^{+\infty} \frac{d \omega}{2 \pi} e^{i \omega 0^{+}} G_{\psi / \chi}(\omega) \partial_{\omega} \tilde{\Sigma}_{\psi / \chi}^{A / B}(\omega)
$$

for two fermions $\psi, \chi$ in both model $A$ and model $B$. To do so, we will use the properties of the spectral functions

$$
\mathscr{A}_{\psi}(\omega)=\frac{C_{\psi}}{\pi} \frac{S_{\psi, \pm}}{|\omega|^{1-2 \Delta_{\psi}}}, \quad \mathscr{A}_{\chi}(\omega)=\frac{C_{\chi}}{\pi} \frac{S_{\chi, \pm}}{|\omega|^{1-2 \Delta_{\chi}}},
$$

where the notation $S_{ \pm}$stands for $S_{ \pm}=\sin (\pi \Delta \pm \theta)$, and \pm depends on the sign of $\omega$. Our convention here is

$$
\mathscr{A}(\omega)=\mp \frac{1}{\pi} \operatorname{Im} G^{R / A}(\omega), \quad G(z)=\int_{-\infty}^{+\infty} d \omega \frac{\mathscr{A}(\omega)}{z-\omega} .
$$

a. Model $A$ Using the melonic S-D equation, we obtain the Fourier transformation of $\tilde{\Sigma}_{\psi}^{A}(\tau)$

$$
\begin{aligned}
\tilde{\Sigma}_{\psi}^{A}(\omega) & =-4 g^{2} \sqrt{\frac{M_{2}}{M_{1}}} \int_{-\infty}^{+\infty} \frac{d \nu_{1}}{2 \pi} \frac{d \nu_{2}}{2 \pi} \frac{d \nu_{3}}{2 \pi} G_{\psi}\left(\nu_{1}\right) G_{\chi}\left(\nu_{2}\right) G_{\chi}\left(\nu_{3}\right) 2 \pi \delta\left(\nu_{1}+\nu_{2}-\nu_{3}-\omega\right) \\
& =-4 g^{2} \sqrt{\frac{M_{2}}{M_{1}}} \int_{\left\{\omega_{1}^{+}, \omega_{2}^{+}, \omega_{3}^{-}\right\} \cup\left\{\omega_{1}^{-}, \omega_{2}^{-}, \omega_{3}^{+}\right\}} d \omega_{1} d \omega_{2} d \omega_{3} \frac{\mathscr{A}_{\psi}\left(\omega_{1}\right) \mathscr{A}_{\chi}\left(\omega_{2}\right) \mathscr{A}_{\chi}\left(\omega_{3}\right)}{\omega_{1}+\omega_{2}-\omega-\omega_{3}+i 0^{+} \operatorname{sgn}\left(\omega_{3}\right)},
\end{aligned}
$$

where the notation $\left\{\omega_{1}^{+}, \omega_{2}^{+}, \omega_{3}^{-}\right\}$means the integration domain $\left\{\omega_{1}>0, \omega_{2}>0, \omega_{3}<0\right\}$. Accordingly, the integral Eq. B4 for $\psi$ reads

$$
\begin{aligned}
\mathcal{I}_{\psi}^{A} & =i \mathbb{P} \int_{-\infty}^{+\infty} \frac{d \omega d \omega_{0}}{2 \pi} \frac{\mathscr{A}_{\psi}\left(\omega_{0}\right) e^{i \omega 0^{+}}}{\omega-\omega_{0}+i 0^{+} \operatorname{sgn}\left(\omega_{0}\right)} \partial_{\omega} \tilde{\Sigma}_{\psi}^{A}(\omega) \\
& =\frac{4 g^{2}}{2 \pi i} \sqrt{\frac{M_{2}}{M_{1}}} \int_{\Gamma} d \omega_{0} d \omega_{1} d \omega_{2} d \omega_{3} \mathscr{A}_{\psi}\left(\omega_{0}\right) \mathscr{A}_{\psi}\left(\omega_{1}\right) \mathscr{A}_{\chi}\left(\omega_{2}\right) \mathscr{A}_{\chi}\left(\omega_{3}\right) \Phi_{\delta}\left(\omega_{1}+\omega_{2}-\omega_{3}-i 0^{+} \operatorname{sgn} \omega_{1}, \omega_{0}-i 0^{+} \operatorname{sgn} \omega_{0}\right)
\end{aligned}
$$

The integration domain of $\mathcal{I}_{\psi}^{A}$ is $\Gamma=\Gamma_{1} \cup \Gamma_{2} \cup \Gamma_{3} \cup \Gamma_{4}$ where

$$
\Gamma_{1}=\left\{\omega_{0}^{+}, \omega_{1}^{+}, \omega_{2}^{+}, \omega_{3}^{-}\right\}, \quad \Gamma_{2}=\left\{\omega_{0}^{-}, \omega_{1}^{+}, \omega_{2}^{+}, \omega_{3}^{-}\right\}, \quad \Gamma_{3}=\left\{\omega_{0}^{+}, \omega_{1}^{-}, \omega_{2}^{-}, \omega_{3}^{+}\right\}, \quad \Gamma_{4}=\left\{\omega_{0}^{-}, \omega_{1}^{-}, \omega_{2}^{-}, \omega_{3}^{+}\right\} .
$$


We have also used the function

$$
\Phi_{\delta}\left(a+i \epsilon_{a}, b+i \epsilon_{b}\right)=\mathbb{P} \int_{-\infty}^{+\infty} d z \frac{e^{i \omega 0^{+}}}{\left(z-a-i \epsilon_{a}\right)^{2}\left(z-b-i \epsilon_{b}\right)}
$$

where $a, b \in \mathbb{R}$ and $\epsilon_{a}, \epsilon_{b} \rightarrow 0$. The expression of $\Phi_{\delta}$ is explicitly calculated as Eq. A8 in Ref. 50. In the following, we will only use its property $\Phi_{\delta}\left(-a-i \epsilon_{a},-b-i \epsilon_{b}\right)=-\Phi_{\delta}\left(a+i \epsilon_{a}, b+i \epsilon_{b}\right)$. By changing of variables, we could write the integral as

$$
\mathcal{I}_{\psi}^{A}=\frac{4 g^{2}}{2 \pi i} \sqrt{\frac{M_{2}}{M_{1}}} \int_{x_{i}>0} \prod_{i=0}^{3} d x_{i}\left(\begin{array}{r}
\left(\mathscr{A}_{\psi}\left(x_{1}\right) \mathscr{A}_{\chi}\left(x_{2}\right) \mathscr{A}_{\chi}\left(-x_{3}\right) \mathscr{A}_{\psi}\left(-x_{0}\right)-\mathscr{A}_{\psi}\left(-x_{1}\right) \mathscr{A}_{\chi}\left(-x_{2}\right) \mathscr{A}_{\chi}\left(x_{3}\right) \mathscr{A}_{\psi}\left(x_{0}\right)\right) \\
\times \Phi_{\delta}\left(x_{1}+x_{2}+x_{3}-i \epsilon_{1},-x_{0}+i \epsilon_{0}\right)+ \\
\left(\mathscr{A}_{\psi}\left(x_{1}\right) \mathscr{A}_{\chi}\left(x_{2}\right) \mathscr{A}_{\chi}\left(-x_{3}\right) \mathscr{A}_{\psi}\left(x_{0}\right)-\mathscr{A}_{\psi}\left(-x_{1}\right) \mathscr{A}_{\chi}\left(-x_{2}\right) \mathscr{A}_{\chi}\left(x_{3}\right) \mathscr{A}_{\psi}\left(-x_{0}\right)\right) \\
\times \Phi_{\delta}\left(x_{1}+x_{2}+x_{3}-i \epsilon_{1}, x_{0}-i \epsilon_{0}\right)
\end{array}\right) .
$$

Using the expressions Eq. B5, we have

$$
\begin{aligned}
& \mathscr{A}_{\psi}\left(x_{1}\right) \mathscr{A}_{\chi}\left(x_{2}\right) \mathscr{A}_{\chi}\left(-x_{3}\right) \mathscr{A}_{\psi}\left(-x_{0}\right)-\mathscr{A}_{\psi}\left(-x_{1}\right) \mathscr{A}_{\chi}\left(-x_{2}\right) \mathscr{A}_{\chi}\left(x_{3}\right) \mathscr{A}_{\psi}\left(x_{0}\right) \\
= & \frac{C_{\psi}^{2} C_{\chi}^{2}}{\pi^{4}} \frac{S_{\psi,+} S_{\chi,+} S_{\chi,-} S_{\psi,-}-S_{\psi,-} S_{\chi,-} S_{\chi,+} S_{\psi,+}}{\left|x_{0}\right|^{1-2 \Delta_{\psi}}\left|x_{1}\right|^{1-2 \Delta_{\psi}}\left|x_{2}\right|^{1-2 \Delta_{\chi}}\left|x_{3}\right|^{1-2 \Delta_{\chi}}}=0 .
\end{aligned}
$$

Thus, the first term vanishes, and we only need to calculate the second term

$$
\mathcal{I}_{\psi}^{A}=\frac{4 g^{2}}{2 \pi i} \sqrt{\frac{M_{2}}{M_{1}}} \frac{C_{\psi}^{2}}{\pi^{4}} \int_{u_{i}>0}^{2} \prod_{i=0}^{3} d u_{i} \frac{S_{\psi,+}^{2} S_{\chi,+} S_{\chi,-}-S_{\psi,-}^{2} S_{\chi,-} S_{\chi,+}}{\left|u_{0} u_{1}\right|^{1-2 \Delta_{\psi}}\left|u_{2} u_{3}\right|^{1-2 \Delta_{\chi}}} \Phi_{\delta=1}\left(u_{1}+u_{2}+u_{3}-i \epsilon_{1}, u_{0}-i \epsilon_{0}\right),
$$

where we have introduced new variables $x_{i}=u_{i} \delta$ to take the limit $\delta \rightarrow 0^{+}$.

Before calculating the integral, we want to show $\mathcal{I}_{\psi}^{A}$ does not depend on $M_{1}, M_{2}$. On one hand, the straightforward calculation gives

$$
S_{\psi,+}^{2} S_{\chi,+} S_{\chi,-}-S_{\psi,-}^{2} S_{\chi,-} S_{\chi,+}=\frac{1}{2} \sin \left(2 \pi \Delta_{\psi}\right) \sin \left(2 \theta_{\psi}\right)\left(\cos \left(2 \theta_{\chi}\right)-\cos \left(2 \pi \Delta_{\chi}\right)\right) .
$$

On the other hand, we read from the S-D equation

$$
C_{\psi}^{2} C_{\chi}^{2}=\frac{1}{2 g^{2}} \sqrt{\frac{M_{1}}{M_{2}}} \frac{\pi\left(1-2 \Delta_{\psi}\right) \sin \left(2 \pi \Delta_{\psi}\right)}{\cos \left(2 \pi \Delta_{\psi}\right)+\cos \left(2 \theta_{\chi}\right)} .
$$

They together give us

$$
\mathcal{I}_{\psi}^{A}=\frac{1}{i \pi^{4}} F^{A}\left(\Delta_{\psi}\right)\left(\frac{1}{2}-\Delta_{\psi}\right) \sin ^{2}\left(2 \pi \Delta_{\psi}\right) \sin \left(2 \theta_{\psi}\right)
$$

where

$$
F^{A}\left(\Delta_{\psi}\right)=\int_{u_{i}>0} \prod_{i=0}^{3} d u_{i} \frac{\Phi_{\delta=1}\left(u_{1}+u_{2}+u_{3}-i \epsilon_{1}, u_{0}-i \epsilon_{0}\right)}{\left|u_{0} u_{1}\right|^{1-2 \Delta_{\psi}}\left|u_{2} u_{3}\right|^{2 \Delta_{\psi}}} .
$$

Then we define $x=u_{0}, y=u_{1}+u_{2}+u_{3}$, and integrate over $u_{2}, u_{3}$. The result is

$$
F\left(\Delta_{\psi}\right)=\frac{\pi}{\left(1-2 \Delta_{\psi}\right) \sin \left(2 \pi \Delta_{\psi}\right)} \int_{0}^{\infty} d x d y\left(\frac{y}{x}\right)^{1-2 \Delta_{\psi}} \Phi_{\delta=1}\left(y-i \epsilon_{1}, x-i \epsilon_{0}\right) .
$$

We proceed to calculate the integral in the following way

$$
\begin{aligned}
\int_{0}^{\infty} d x d y\left(\frac{y}{x}\right)^{1-2 \Delta_{\psi}} \Phi_{\delta=1}\left(y-i \epsilon_{1}, x-i \epsilon_{0}\right) & =\int_{0}^{\infty} d x d y\left(\frac{y}{x}\right)^{1-2 \Delta_{\psi}} \mathbb{P}_{\delta=1} \int_{-\infty}^{+\infty} d z \frac{e^{i \omega 0^{+}}}{\left(z-y+i \epsilon_{1}\right)^{2}\left(z-x+i \epsilon_{0}\right)} \\
& =\pi^{2} \frac{\left(1-2 \Delta_{\psi}\right)}{\sin ^{2}\left(2 \pi \Delta_{\psi}\right)} \mathbb{P}_{\delta=1} \int_{-\infty}^{+\infty} d z \frac{e^{i z 0^{+}}}{z}=i \pi^{3} \frac{\left(1-2 \Delta_{\psi}\right)}{\sin ^{2}\left(2 \pi \Delta_{\psi}\right)}
\end{aligned}
$$


Thus, we have

$$
F\left(\Delta_{\psi}\right)=\frac{i \pi^{4}}{\sin ^{3}\left(2 \pi \Delta_{\psi}\right)} \Longrightarrow \mathcal{I}_{\psi}=\left(\frac{1}{2}-\Delta_{\psi}\right) \frac{\sin \left(2 \theta_{\psi}\right)}{\sin \left(2 \pi \Delta_{\psi}\right)}
$$

In conclusion, we arrive at the result Eq. 25, which is consistent with the expression $\mathcal{Q}(\theta, \Delta)$ in $\operatorname{Ref} 39$ for the complex $\mathrm{SYK}_{q}$ model with the conformal dimension $\Delta=1 / q$.

Through similar calculations based on

$$
\mathcal{I}_{\chi}^{A}=\frac{4 g^{2}}{2 \pi i} \sqrt{\frac{M_{1}}{M_{2}}} \frac{C_{\psi}^{2} C_{\chi}^{2}}{\pi^{4}} \int_{u_{i}>0} \prod_{i=0}^{3} d u_{i} \frac{S_{\chi,+}^{2} S_{\psi,+} S_{\psi,-}-S_{\chi,-}^{2} S_{\psi,-} S_{\psi,+}}{\left|u_{0} u_{1}\right|^{1-2 \Delta_{\psi}}\left|u_{2} u_{3}\right|^{1-2 \Delta_{\chi}}} \Phi_{\delta=1}\left(u_{1}+u_{2}+u_{3}-i \epsilon_{1}, u_{0}-i \epsilon_{0}\right)
$$

we obtain the identical expression Eq. 26 for $\chi$ fermion. In model $A, \theta_{\psi}, \theta_{\chi}$ are two independent variables, and U (1) charges for $\psi, \chi$ are conserved separately.

b. Model $B$ The expression of $\tilde{\Sigma}^{B}$ is a bit different from $\tilde{\Sigma}^{A}$

$$
\begin{aligned}
\tilde{\Sigma}_{\psi}^{B}(\omega) & =-4 g^{2} \sqrt{\frac{M_{2}}{M_{1}}} \int_{-\infty}^{+\infty} \frac{d \nu_{1}}{2 \pi} \frac{d \nu_{2}}{2 \pi} \frac{d \nu_{3}}{2 \pi} G_{\chi}\left(\nu_{1}\right) G_{\chi}\left(\nu_{2}\right) G_{\psi}\left(\nu_{3}\right) 2 \pi \delta\left(\nu_{1}+\nu_{2}-\nu_{3}-\omega\right) \\
& =-4 g^{2} \sqrt{\frac{M_{2}}{M_{1}}} \int_{\left\{\omega_{1}^{+}, \omega_{2}^{+}, \omega_{3}^{-}\right\} \cup\left\{\omega_{1}^{-}, \omega_{2}^{-}, \omega_{3}^{+}\right\}} d \omega_{1} d \omega_{2} d \omega_{3} \frac{\mathscr{A}_{\chi}\left(\omega_{1}\right) \mathscr{A}_{\chi}\left(\omega_{2}\right) \mathscr{A}_{\psi}\left(\omega_{3}\right)}{\omega_{1}+\omega_{2}-\omega-\omega_{3}+i 0^{+} \operatorname{sgn}\left(\omega_{3}\right)} .
\end{aligned}
$$

Now the integral Eq. B4 for $\psi$ reads

$$
\mathcal{I}_{\psi}^{B}=\frac{4 g^{2}}{2 \pi i} \sqrt{\frac{M_{2}}{M_{1}}} \int_{\Gamma} d \omega_{0} d \omega_{1} d \omega_{2} d \omega_{3} \mathscr{A}_{\psi}\left(\omega_{0}\right) \mathscr{A}_{\chi}\left(\omega_{1}\right) \mathscr{A}_{\chi}\left(\omega_{2}\right) \mathscr{A}_{\psi}\left(\omega_{3}\right) \Phi_{\delta}\left(\omega_{1}+\omega_{2}-\omega_{3}-i 0^{+} \operatorname{sgn} \omega_{1}, \omega_{0}-i 0^{+} \operatorname{sgn} \omega_{0}\right)
$$

with the same integration domain as $\mathcal{I}_{\psi}^{A}$. By changing of variables, we could write the integral as

$$
\mathcal{I}_{\psi}^{B}=\frac{4 g^{2}}{2 \pi i} \sqrt{\frac{M_{2}}{M_{1}}} \int_{x_{i}>0} \prod_{i=0}^{3} d x_{i}\left(\begin{array}{c}
\left(\mathscr{A}_{\chi}\left(x_{1}\right) \mathscr{A}_{\chi}\left(x_{2}\right) \mathscr{A}_{\psi}\left(-x_{3}\right) \mathscr{A}_{\psi}\left(-x_{0}\right)-\mathscr{A}_{\chi}\left(-x_{1}\right) \mathscr{A}_{\chi}\left(-x_{2}\right) \mathscr{A}_{\psi}\left(x_{3}\right) \mathscr{A}_{\psi}\left(x_{0}\right)\right) \\
\times \Phi_{\delta}\left(x_{1}+x_{2}+x_{3}-i \epsilon_{1},-x_{0}+i \epsilon_{0}\right)+ \\
\left(\mathscr{A}_{\chi}\left(x_{1}\right) \mathscr{A}_{\chi}\left(x_{2}\right) \mathscr{A}_{\psi}\left(-x_{3}\right) \mathscr{A}_{\psi}\left(x_{0}\right)-\mathscr{A}_{\chi}\left(-x_{1}\right) \mathscr{A}_{\chi}\left(-x_{2}\right) \mathscr{A}_{\psi}\left(x_{3}\right) \mathscr{A}_{\psi}\left(-x_{0}\right)\right) \\
\times \Phi_{\delta}\left(x_{1}+x_{2}+x_{3}-i \epsilon_{1}, x_{0}-i \epsilon_{0}\right)
\end{array}\right)
$$

Using the expressions Eq. B5, we have

$$
\begin{aligned}
& \mathscr{A}_{\chi}\left(x_{1}\right) \mathscr{A}_{\chi}\left(x_{2}\right) \mathscr{A}_{\psi}\left(-x_{3}\right) \mathscr{A}_{\psi}\left(-x_{0}\right)-\mathscr{A}_{\chi}\left(-x_{1}\right) \mathscr{A}_{\chi}\left(-x_{2}\right) \mathscr{A}_{\psi}\left(x_{3}\right) \mathscr{A}_{\psi}\left(x_{0}\right) \\
= & \frac{C_{\psi}^{2} C_{\chi}^{2}}{\pi^{4}} \frac{S_{\chi,+} S_{\chi,+} S_{\psi,-} S_{\psi,-}-S_{\chi,-} S_{\chi,-} S_{\psi,+} S_{\psi,+}}{\left|x_{0}\right|^{1-2 \Delta_{\psi}}\left|x_{1}\right|^{1-2 \Delta_{\chi}}\left|x_{2}\right|^{1-2 \Delta_{\chi}}\left|x_{3}\right|^{1-2 \Delta_{\psi}}},
\end{aligned}
$$

which seems nonzero at first glance. But it indeed vanishes due to the constraint Eq. 37, and we only need to calculate the second term

$$
\mathcal{I}_{\psi}^{B}=\frac{4 g^{2}}{2 \pi i} \sqrt{\frac{M_{2}}{M_{1}}} \frac{C_{\psi}^{2} C_{\chi}^{2}}{\pi^{4}} \int_{u_{i}>0} \prod_{i=0}^{3} d u_{i} \frac{S_{\chi,+}^{2} S_{\psi,-} S_{\psi,+}-S_{\chi,-}^{2} S_{\psi,+} S_{\psi,-}}{\left|u_{0} u_{3}\right|^{1-2 \Delta_{\psi}}\left|u_{1} u_{2}\right|^{1-2 \Delta_{\chi}}} \Phi_{\delta=1}\left(u_{1}+u_{2}+u_{3}-i \epsilon_{1}, u_{0}-i \epsilon_{0}\right)
$$

where we have again used new variables $x_{i}=u_{i} \delta$. We proceed to analyze the coefficient. The straightforward calculation gives

$$
S_{\chi,+}^{2} S_{\psi,+} S_{\psi,-}-S_{\chi,-}^{2} S_{\psi,-} S_{\psi,+}=\frac{1}{2} \sin \left(2 \pi \Delta_{\chi}\right) \sin \left(2 \theta_{\chi}\right)\left(\cos \left(2 \theta_{\psi}\right)-\cos \left(2 \pi \Delta_{\psi}\right)\right) .
$$

By using the expression Eq. B18 of $C_{\psi}^{2} C_{\chi}^{2}$ and the constraint Eq. 37, we are able to obtain a similar form comparing to Eq. B19

$$
\mathcal{I}_{\psi}^{B}=\frac{1}{i \pi^{4}} F^{B}\left(\Delta_{\psi}\right)\left(\frac{1}{2}-\Delta_{\psi}\right) \sin ^{2}\left(2 \pi \Delta_{\psi}\right) \sin \left(2 \theta_{\psi}\right)
$$


where

$$
F^{B}\left(\Delta_{\psi}\right)=\int_{u_{i}>0} \prod_{i=0}^{3} d u_{i} \frac{\Phi_{\delta=1}\left(u_{1}+u_{2}+u_{3}-i \epsilon_{1}, u_{0}-i \epsilon_{0}\right)}{\left|u_{0} u_{3}\right|^{1-2 \Delta_{\psi}}\left|u_{1} u_{2}\right|^{2 \Delta_{\psi}}} .
$$

The definition of $F^{B}(\Delta)$ here differs from $F^{A}(\Delta)$ by exchanging $u_{1} \leftrightarrow u_{3}$. Notice that $\epsilon_{1}=-\epsilon_{3}$ which makes the definition looks nonequivalent. However, after defining $x=u_{0}, y=u_{1}+u_{2}+u_{3}$, and integrating over $u_{2}$, $u_{3}$, we still have the expression Eq. B21. Thus, we have exactly the same result Eq. 25 for $\left\langle\mathcal{Q}_{\psi}^{B}\right\rangle$.

Through similar calculations for $\chi$ fermion

$$
\mathcal{I}_{\chi}^{B}=\frac{4 g^{2}}{2 \pi i} \sqrt{\frac{M_{1}}{M_{2}}} \frac{C_{\psi}^{2} C_{\chi}^{2}}{\pi^{4}} \int_{u_{i}>0} \prod_{i=0}^{3} d u_{i} \frac{S_{\psi,+}^{2} S_{\chi,-} S_{\chi,+}-S_{\psi,-}^{2} S_{\chi,+} S_{\chi,-}}{\left|u_{0} u_{3}\right|^{1-2 \Delta_{\psi}}\left|u_{1} u_{2}\right|^{1-2 \Delta_{\chi}}} \Phi_{\delta=1}\left(u_{1}+u_{2}+u_{3}-i \epsilon_{1}, u_{0}-i \epsilon_{0}\right),
$$

we again obtain exactly the same expression Eq. 26 for $\mathcal{Q}_{\chi}^{B}$. Despite the similarity in expressions, only the total U (1) charge filling factor Eq. 34 is a conserved quantity in model B.

1 J. A. Hertz, Phys. Rev. B 14, 1165 (1976), URL https: //link.aps.org/doi/10.1103/PhysRevB.14.1165

2 A. J. Millis, Phys. Rev. B 48, 7183 (1993), URL https: //link.aps.org/doi/10.1103/PhysRevB.48.7183

${ }^{3}$ H. v. Löhneysen, A. Rosch, M. Vojta, and P. Wölfle, Rev. Mod. Phys. 79, 1015 (2007), URL https://link.aps. org/doi/10.1103/RevModPhys.79.1015.

4 J. Polchinski, Nuclear Physics B 422, 617 (1994), ISSN 0550-3213, URL http://www.sciencedirect.com/ science/article/pii/0550321394904499

${ }^{5}$ C. Nayak and F. Wilczek, Nuclear Physics B 417, 359 (1994), ISSN 0550-3213, URL http: //www.sciencedirect.com/science/article/pii/ 0550321394904774

${ }^{6}$ C. Nayak and F. Wilczek, Nuclear Physics B 430, 534 (1994), ISSN 0550-3213, URL http: //www.sciencedirect.com/science/article/pii/ 0550321394901589 .

7 V. Oganesyan, S. A. Kivelson, and E. Fradkin, Phys. Rev. B 64, 195109 (2001), URL https://link.aps.org/doi/ 10.1103/PhysRevB.64.195109.

${ }^{8}$ S.-S. Lee, Phys. Rev. B 80, 165102 (2009), URL https: //link.aps.org/doi/10.1103/PhysRevB.80.165102

9 D. F. Mross, J. McGreevy, H. Liu, and T. Senthil, Phys. Rev. B 82, 045121 (2010), URL https://link.aps.org/ doi/10.1103/PhysRevB.82.045121.

io M. A. Metlitski and S. Sachdev, Phys. Rev. B 82, 075127 (2010), URL https://link.aps.org/doi/10. 1103/PhysRevB.82.075127

${ }^{11}$ M. A. Metlitski and S. Sachdev, Phys. Rev. B 82, 075128 (2010), URL https://link.aps.org/doi/10. 1103/PhysRevB.82.075128

12 A. Schlief, P. Lunts, and S.-S. Lee, Phys. Rev. X 7, 021010 (2017), URL https://link.aps.org/doi/10. 1103/PhysRevX.7.021010.

${ }^{13}$ Y. Schattner, S. Lederer, S. A. Kivelson, and E. Berg, Phys. Rev. X 6, 031028 (2016), URL https://link.aps . org/doi/10.1103/PhysRevX.6.031028.

${ }^{14}$ M. Gurvitch and A. T. Fiory, Phys. Rev. Lett. 59, 1337 (1987), URL https://link.aps.org/doi/10.1103/
PhysRevLett.59.1337

15 S. W. Tozer, A. W. Kleinsasser, T. Penney, D. Kaiser, and F. Holtzberg, Phys. Rev. Lett. 59, 1768 (1987), URL https://link. aps .org/doi/10.1103/PhysRevLett. 59.1768

16 S. Martin, A. T. Fiory, R. M. Fleming, L. F. Schneemeyer, and J. V. Waszczak, Phys. Rev. Lett. 60, 2194 (1988), URL https://link .aps.org/doi/10.1103/PhysRevLett . 60.2194

17 S. Martin, A. T. Fiory, R. M. Fleming, L. F. Schneemeyer, and J. V. Waszczak, Phys. Rev. B 41, 846 (1990), URL https://link.aps.org/doi/10.1103/PhysRevB.41.846.

18 C. M. Varma, P. B. Littlewood, S. Schmitt-Rink, E. Abrahams, and A. E. Ruckenstein, Phys. Rev. Lett. 63, 1996 (1989), URL https://link.aps.org/doi/10.1103/ PhysRevLett.63.1996.

19 Y. Cao, D. Chowdhury, D. Rodan-Legrain, O. RubiesBigordà, K. Watanabe, T. Taniguchi, T. Senthil, and P. Jarillo-Herrero, arXiv:1901.03710 (2019).

20 S. Sachdev and J. Ye, Physical Review Letters 70, 3339 (1993), cond-mat/9212030.

21 A. Kitaev, A simple model of quantum holography, http://online.kitp.ucsb.edu/online/entangled15/ kitaev/,http://online.kitp.ucsb.edu/online/ entangled15/kitaev2/. (2015), Talks at KITP, April 7, 2015 and May 27, 2015.

22 S. Sachdev, Physical Review X 5, 041025 (2015), 1506.05111.

23 J. Polchinski and V. Rosenhaus, Journal of High Energy Physics 4, 1 (2016), 1601.06768.

24 J. Maldacena and D. Stanford, Phys. Rev. D 94, 106002 (2016), 1604.07818.

25 E. Witten, ArXiv e-prints (2016), 1610.09758.

${ }^{26}$ I. R. Klebanov and G. Tarnopolsky, Phys. Rev. D 95, 046004 (2017), URL https://link.aps.org/doi/10. 1103/PhysRevD.95.046004

${ }^{27}$ D. J. Gross and V. Rosenhaus, Journal of High Energy Physics 2017, 93 (2017), ISSN 1029-8479, URL https: //doi.org/10.1007/JHEP02(2017)093.

${ }^{28}$ Z. Bi, C.-M. Jian, Y.-Z. You, K. A. Pawlak, and C. Xu, 
Physical Review B 95, 205105 (2017).

29 Z. Luo, Y.-Z. You, J. Li, C.-M. Jian, D. Lu, C. Xu, B. Zeng, and R. Laflamme, arXiv:1712.06458 (2017).

30 M. A. Metlitski, D. F. Mross, S. Sachdev, and T. Senthil, Phys. Rev. B 91, 115111 (2015), URL https://link.aps . org/doi/10.1103/PhysRevB.91.115111.

31 Y. Wang and A. V. Chubukov, Phys. Rev. B 92, 125108 (2015), URL https://link.aps.org/doi/10. 1103/PhysRevB.92.125108

32 I. Mandal, Phys. Rev. B 94, 115138 (2016), URL https: //link.aps.org/doi/10.1103/PhysRevB.94.115138.

33 S. Lederer, Y. Schattner, E. Berg, and S. A. Kivelson, Phys. Rev. Lett. 114, 097001 (2015), URL https://link. aps.org/doi/10.1103/PhysRevLett.114.097001.

${ }^{4}$ Y. Wang, A. Abanov, B. L. Altshuler, E. A. Yuzbashyan, and A. V. Chubukov, Phys. Rev. Lett. 117, 157001 (2016), URL https://link.aps .org/doi/10.1103/PhysRevLett. 117.157001.

35 S. Lederer, Y. Schattner, E. Berg, and S. A. Kivelson, Proceedings of the National Academy of Sciences 114, 4905 (2017), http://www.pnas.org/content/114/19/4905.full.pdf, URL http://www . pnas.org/content/114/19/4905.abstract.

36 E. Fradkin, S. A. Kivelson, and J. M. Tranquada, Rev. Mod. Phys. 87, 457 (2015), URL https://link.aps.org/ doi/10.1103/RevModPhys.87.457

sr M. Mitrano, A. A. Husain, S. Vig, A. Kogar, M. S. Rak, S. I. Rubeck, J. Schmalian, B. Uchoa, J. Schneeloch, R. Zhong, et al., Proceedings of the National Academy of Sciences 115, 5392 (2018), ISSN 0027-8424, http://www.pnas.org/content/115/21/5392.full.pdf, URL http://www.pnas.org/content/115/21/5392

38 X.-Y. Song, C.-M. Jian, and L. Balents, Phys. Rev. Lett. 119, 216601 (2017), URL https://link.aps.org/doi/ 10.1103/PhysRevLett.119.216601

39 R. A. Davison, W. Fu, A. Georges, Y. Gu, K. Jensen, and S. Sachdev, Phys. Rev. B 95, 155131 (2017), URL https: //link.aps.org/doi/10.1103/PhysRevB.95.155131.

40 A. A. Patel, J. McGreevy, D. P. Arovas, and S. Sachdev, Phys. Rev. X 8, 021049 (2018), URL https://link.aps. org/doi/10.1103/PhysRevX.8.021049.
41 D. Chowdhury, Y. Werman, E. Berg, and T. Senthil, Phys. Rev. X 8, 031024 (2018), URL https://link.aps.org/ doi/10.1103/PhysRevX.8.031024

42 X. Wu, X. Chen, C.-M. Jian, Y.-Z. You, and C. Xu, Phys. Rev. B 98, 165117 (2018), URL https://link.aps.org/ doi/10.1103/PhysRevB.98.165117.

43 R. Gurau, Commun. Math. Phys. 304, 69 (2011).

44 N. D. Mathur, F. M. Grosche, S. R. Julian, I. R. Walker, D. M. Freye, R. K. W. Haselwimmer, and G. G. Lonzarich, Nature 394, 39 (1998).

${ }^{45}$ H. Q. Yuan, F. M. Grosche, M. Deppe, G. Sparn, C. Geibel, and F. Steglich, Phys. Rev. Lett. 96, 047008 (2006), URL https : //link . aps .org/doi/10.1103/PhysRevLett. 96.047008

${ }^{46}$ N. Tsujii, H. Kitazawa, T. Aoyagi, T. Kimura, and G. Kido, Journal of Magnetism and Magnetic Materials 310, 349 (2007), ISSN 0304-8853, proceedings of the 17th International Conference on Magnetism, URL http://www.sciencedirect.com/science/article/ pii/S0304885306012248.

4 H. Yuan and F. Steglich, Physica C: Superconductivity and its Applications 460-462, 141 (2007), ISSN 0921-4534, proceedings of the 8th International Conference on Materials and Mechanisms of Superconductivity and High Temperature Superconductors, URL http://www.sciencedirect.com/science/article/ pii/S0921453407005242.

48 O. Stockert and F. Steglich, Annual Review of Condensed Matter Physics 2, 79 (2011), https://doi.org/10.1146/annurev-conmatphys062910-140546, URL https://doi.org/10.1146/ annurev-conmatphys-062910-140546

${ }^{49}$ H. v. Löhneysen, A. Rosch, M. Vojta, and P. Wölfle, Rev. Mod. Phys. 79, 1015 (2007), URL https://link.aps. org/doi/10.1103/RevModPhys.79.1015.

so A. Georges, O. Parcollet, and S. Sachdev, Phys. Rev. B 63, 134406 (2001), URL https://link .aps.org/doi/10. 1103/PhysRevB.63.134406

${ }^{51}$ S. Dartois, H. Erbin, and S. Mondal, arXiv:1706.00412 (2017). 